\title{
Amygdalectomies et masque laryngé renforcé
}

One hundred children were randomly allocated to receive general anaesthesia for tonsillectomy either through a preformed tracheal tube or a reinforced laryngeal mask. The insertion of both devices was easy but occasionally, with the laryngeal mask, the airway became obstructed during the Boyle-Davis gag insertion. There was more coughing afier intubation (ET 26\%, $R L M 0 \%, P<0,001$ ) and extubation (ET 34\%, RLM 0\%, $P<0,001$ ), than after insertion and withdrawal of the $R L M$. Oxygen desaturation (ET 14\%, RLM 2\%,P<0,02) and internal contamination with blood seepage (ET 30\%, RLM 4\%, $P<0,001)$ occurred less frequently with the $R L M$.

Deux séries de 50 enfants devant subir une amygdalectomie et dont la liberté des voies aériennes a été assurée après tirage au sort par une sonde endotrachéale préformée (SE) ou un masque laryngé renforcé (MLR), ont été comparées. L'insertion des deux dispositifs se fait avec la même facilité mais lintroduction de l'ouvre-bouche peut mobiliser le masque et nécessiter sa reposition. Lintubation ( $S E 26 \%, M L 0 \%, P<0,001$ ) et l'extubation (SE 34\%,ML 0\%, $P<0,001$ ) provoquent des secousses de toux que le masque ne déclenche pas. La désaturation pastopératoire ( $S E 14 \%, M L 2 \%, P<0,02$ ) et la contamination des voies aériennes (SE 30\%, ML 4\%,P<0,001) sont plus rares avec le masque laryngé.

Le contrôle des voies aériennes et leur protection, indispensables lors de chirurgie endobuccale, sont les deux impératifs de l'anesthésie pour amygdalectomie. L'intu

\section{Key words}

EQUPMENT: reinforced laryngeal mask airway;

INTUBATION: complications;

COMPLICATIONS: airway obstruction, coughing, contamination.

Du Service d'Anesthesie-Réanimation Chirurgicale (Pr. MarieClaire Laxenaire), CHU Hôpital Central, Nancy, France. Adresser la correspondance à: Dr Dominique BoissonBertrand, Service d'Anesthésie-Réanimation Chirurgicale, CHU Hôpital Central, Case Officielle n 34, F-54035 NancyCedex, France. Accepté pour publication le 15 mai, 1995. bation trachéale permet d'y satisfaire, ${ }^{1}$ mais elle est souvent à l'origine de toux, spasme et oedème de glotte. Lorsque la sonde n'est pas armée, elle peut être écrasée sur les dents par la spatule de l'ouvre-bouche de BoyleDavis, et si elle est préformée, elle peut dans $20 \%$ des cas, être responsable d'une intubation sélective. ${ }^{2}$ Le masque laryngé (ML) a été utilisé par de nombreux auteurs en chirurgie endobuccale ORL, ${ }^{3,4}$ mais son tube préformé est très volumineux dans la cavité buccale d'un enfant, surtout si celui-ci a moins de quatre ans et gêne l'exposition du pharynx. ${ }^{5-8}$ Le masque laryngé renforcé (MLR) ${ }^{9,10}$ se différencie du masque classique, car son tube est armé. Il peut être tourné sur lui-même sans se couder, et risque moins d'être écrasé sur les dents par l'abaisse-langue de l'ouvre-bouche. ${ }^{5-8} \mathrm{Ce}$ tube, souple, peut être mobilisé latéralement sans désolidariser le masque du larynx. Enfin, grâce à son petit diamètre, il se glisse parfaitement dans la gouttière de l'ouvre-bouche de Boyle-Davis qui le maintient fermement en place. ${ }^{8}$

Pour définir les avantages éventuels de ce masque par rapport à la sonde d'intubation préformée dans les amygdalectomies, nous avons comparé deux séries de 50 enfants ASA I, âgés de deux à dix ans, dont la liberté des voies aériennes a été assurée par l'un ou l'autre de ces dispositifs, après tirage au sort grâce à des enveloppes de randomisation scellées. Le consentement éclairé des parents et l'accord du comité d'éthique ont été obtenus.

\section{Méthodes}

Après une prémédication de $60 \mu \mathrm{g} \cdot \mathrm{kg}^{-1}$ de flunitrazépam intrarectal, l'anesthésie a été induite par le même médecin, à l'aide de 2,5 à $3 \mathrm{mg} \cdot \mathrm{kg}^{-1}$ de propofol pour les enfants de plus de huit ans ou pesant plus de $20 \mathrm{~kg}$ (ce qui représente $40 \%$ des enfants de chaque série), pour les autres avec de l'halothane (1,5 à 2 vol\%) conservé pour l'entretien de la narcose dans tous les cas. Tous les enfants ont été perfusés. L'analgésie a été assurée si nécessaire, lorsque la fréquence cardiaque dépassait 100 batt $\cdot \min ^{-1}$, par l'injection de 50 à $100 \mu \mathrm{g}$ d'alfentanil. Dès que la pupille était en position centrale et fixe, ont été mis en place, sans adjonction de curare, soit un masque laryngé renforcé Intavent de taille 2-2,5 ou 3 selon le poids de l'enfant, soit une sonde dintubation Mallinckrodt préformée, sans ballonnet, de taille 3,5 à 6,5 , 
selon le diamètre de la glotte. Les sondes sans ballonnet étant recommandées chez l'enfant de moins de six ans, leur utilisation a été étendue à tous afin de conserver l'homogénéité de la série.

Le MLR se place comme le masque classique après avoir soigneusement dégonflé le coussinet. Un peu d'eau suffit à le lubrifier. Après avoir mis la tête de l'enfant en hyperextension et bien appliqué le masque derrière les incisives supérieures, en le tenant comme un crayon à la jonction du coussinet et du tube, le masque est poussé avec l'index tendu le long du palais dur, le plus loin possible, car la souplesse de son tube ne permet pas de le guider aussi facilement que le masque classique. L'insufflation de 10 à $20 \mathrm{ml}$ d'air, selon la taille du coussinet, assure l'étanchéité de la jonction avec le larynx et place le masque en position définitive. Il peut alors être fixé sur la lèvre inférieure.

Les patients respiraient spontanément un mélange à part égale d'oxygène et de protoxyde d'azote, dans lequel était introduit l'halothane nécessaire au maintien de la narcose. Ils ventilaient à travers une valve de Digby-Leigh qui permettait d'assister manuellement la ventilation si la $\mathrm{FETCO}_{2}$ était supérieure à 6 vol.\%, sans gêner le chirurgien placé à la tête. Cette assistance ventilatoire, sans fuite, est possible avec le MLR si la pression d'insufflation est inférieure à $20 \mathrm{~cm} \mathrm{l} \mathrm{H}_{2} \mathrm{O}$."

Le nombre de tentatives dintroduction du masque ou de la sonde avant leur mise en place définitive a été évalué, ainsi que les déplacements éventuels du MLR lors de l'introduction de l'ouvre-bouche par l'opérateur. La visibilité du champ opératoire a été jugée satisfaisante ou non par le chirurgien qui ignorait le type de matériel utilisé. Les réactions de l'enfant à la pose ou au retrait du dispositif (toux, spasme, désaturation oxyhémoglobinée) ont été notées. Les valeurs des paramètres hémodynamiques (fc, $\mathrm{Pa}_{\text {sys }}, \mathrm{Pa}_{\mathrm{dia}}$ ) et respiratoires $\left(\mathrm{SpO}_{2}\right.$, FErCO ${ }_{2}$ et FI halothane) ont été relevées toutes les dix minutes.

Pour évaluer la contamination éventuelle des voies aériennes par le sang, ont été examinées à leur retrait la face laryngée du MLR, et la paroi transparente de la sonde de Mallinckrodt après qu'elle ait été soigneusement essuyé, pour ne conserver que sa contamination interne. Une fibroscopie (fibroscope Olympus enfant) a été réalisée au travers du masque laryngé chez cinq enfants choisis au hasard afin de contrôler l'efficacité de la protection des voies aériennes. Le masque laryngé ou la sonde ont été retirés en fin dintervention dès la réapparition du réflexe photomoteur, les enfants étant encore endormis, ${ }^{12}$ après aspiration soigneuse de la cavité buccale et vérification de l'absence de saignement. Le masque laryngé a été retiré gonflé, comme il est conseillé lorsque le patient n'est pas réveillé.
Les résultats, exprimés en moyenne assortie de son écart-type, ont été comparés à l'aide du test $t$ de Student pour les variables quantitatives, et du test $\mathrm{du}_{\mathrm{Chi}}{ }^{2}$ pour les variables qualitatives.

\section{Résultats}

Les amygdalectomies par dissection, associées souvent à l'ablation des végétations adénoïdes, parfois à une paracentèse ou une pose de drains transtympaniques ont été réalisées par différents chirurgiens qui ont opéré le même nombre d'enfants dans chaque série. Les deux séries sont homogènes en ce qui concerne l'âge, le sexe, le poids des enfants, la durée d'intervention. La comparaison des moyennes des paramètres hémodynamiques et ventilatoires (Tableau I), montre que les deux séries ne sont pas significativement différentes. L'assistance manuelle de la ventilation et la prescription d'analgésiques n'ont pas été différentes dans les deux séries. La visibilité du champ opératoire a été parfaite dans tous les cas et les deux dispositifs très bien tolérés par les patients.

La mise en place de la sonde d'intubation ou du masque laryngé s'est faite avec la même facilité. Pour l'intubation, dans deux cas, deux et quatre tentatives ont été nécessaires, la glotte étant particulièrement haut située. Pour le masque laryngé dans un cas, deux essais ont été nécessaires, et dans un autre la mise en place a été impossible et la trachée a dû être intubée, ce qui l'a fait passer dans l'autre groupe. Les tailles des différents dispositifs sont regroupées dans le Tableau II. Lintroduction de l'ouvre-bouche de Boyle-Davis a entravé la ventilation dans dix cas, ce qui a nécessité sa remise en place, parfois associée à celle du MLR, ceci ne s'est jamais produit avec la sonde, et l'intubation n'a jamais été sélective.

Lintubation a provoqué des secousses de toux dans 26\% des cas (Tableau III), suivies de spasmes dans trois cas, dont un a provoqué une désaturation à $77 \%$. Deux fois la pose du masque laryngé a déclenché un stridor rapidement résolutif à l'approfondissement de l'anesthésie, dont l'un s'est également accompagné de désaturation $\left(\mathrm{SpO}_{2}\right.$ à $\left.70 \%\right)$. A l'extubation ces phénomènes ont été encore plus fréquents, puisque $34 \%$ des patients intubés ont toussé, déclenchant un spasme de la glotte dans 7 des 17 cas. Douze pour cent des enfants ont présenté un épisode de désaturation importante $\left(\mathrm{SpO}_{2} \leq 85 \%\right)$, toujours en rapport avec un spasme de la glotte, qu'il soit ou non précédé de toux. Cette toux ne s'est pas produite chez les opérés porteurs dun MLR. Néanmoins cinq d'entre eux ont présenté un spasme de la glotte, provoquant une désaturation dans un seul cas $\left(\mathrm{SpO}_{2}\right.$ à $85 \%)$.

Les fibroscopies postopératoires réalisées chez cinq enfants au travers du masque laryngé n'ont pas montré la 
TABLEAU I Caractères anthropométriques, cardio-vasculaires et respiratoires avec masque laryngé renforcé (MLR) et sonde endotrachéale (SE)

\begin{tabular}{lcc}
\hline & $\begin{array}{c}\text { Masque laryngé } \\
\text { renforcé } \\
(n=50)\end{array}$ & $\begin{array}{l}\text { Sonde } \\
\text { endotrachéale } \\
(n=50)\end{array}$ \\
\hline Patients (garçons/filles) & $22 / 28$ & $23 / 27$ \\
Age (ans) & $5 \pm 2$ & $5 \pm 3$ \\
Poids (kg) & $19 \pm 7$ & $21 \pm 9$ \\
Durée (min) & $41 \pm 18$ & $41 \pm 21$ \\
FC (b· min & $106 \pm 14$ & $105 \pm 23$ \\
$\mathrm{~Pa}_{\text {sys }}$ (mmHg) & $102 \pm 14$ & $98 \pm 13$ \\
$\mathrm{~Pa}_{\text {dia }}(\mathrm{mmHg})$ & $67 \pm 11$ & $61 \pm 13$ \\
$\mathrm{FETCO}_{2}(\%)$ & $5,5 \pm 0,8$ & $5,5 \pm 0,8$ \\
$\mathrm{FET} \mathrm{halothane}(\%)_{\mathrm{SaO}_{2}(\%)}^{1,34 \pm 0,50}$ & $1,46 \pm 0,22$ \\
\hline
\end{tabular}

Moyenne \pm écart-type de la moyenne.

Pas de diffférence significative.

TABLEAU II Differents dispositifs de ventilation utilisés

\begin{tabular}{|c|c|c|c|}
\hline \multicolumn{2}{|c|}{ Masque laryngé renforcé } & \multicolumn{2}{|c|}{ Sonde endotrachéale } \\
\hline Taille & Patients & Taille & Patients \\
\hline \multirow[t]{2}{*}{2} & 46 & 3,5 & 1 \\
\hline & & 4 & 1 \\
\hline \multirow[t]{2}{*}{2,5} & 2 & 4,5 & 5 \\
\hline & & 5 & 18 \\
\hline \multirow[t]{3}{*}{3} & 2 & 5,5 & 10 \\
\hline & & 6 & 12 \\
\hline & & 6,5 & 3 \\
\hline
\end{tabular}

présence de sang dans les voies respiratoires, même au delà de la glotte. Ceci a pu être vérifié plus simplement en regardant la face endolaryngée du masque, dans deux cas elle s'est trouvée légèrement souillée, tandis que la contamination interne de la sonde endotrachéale, constatée au travers de sa paroi transparente, s'est produite dans 15 cas sur 50 (Tableau III).

\section{Discussion}

La plupart des auteurs trouvent le masque laryngé classique plus facile à insérer qu'une sonde endotrachéale, car il évite la laryngoscopie. ${ }^{13-15}$ Néanmoins le masque laryngé renforcé est plus délicat à mettre en place que le masque classique en raison de la souplesse de son tube armé $^{5-7}$ et la technique habituelle est parfois insuffisante lorsqu'une grosse base de langue ou des amygdales hypertrophiques gênent sa progression. Un coussin placé sous la tête en modifiant l'angle du rachis ou la luxation de la mandibule en soulevant la base de langue, peuvent faciliter son insertion. Une bonne application du masque
TABLEAU III Comparaison des incidents survenant à la mise en place et au retrait du masque laryngé renforcé (MLR) et de la sonde endotrachéale (SE)

\begin{tabular}{|c|c|c|c|c|c|}
\hline & \multicolumn{2}{|c|}{$\begin{array}{l}\text { Masque laryngé } \\
\text { renforcé } \\
(n=50)\end{array}$} & \multicolumn{2}{|c|}{$\begin{array}{l}\text { Sonde } \\
\text { endotrachéale } \\
(n=50)\end{array}$} & \multirow[b]{2}{*}{$P$} \\
\hline & $N b$ de cas & $(\%)$ & $N b$ de cas & (\%) & \\
\hline \multicolumn{6}{|l|}{ Mise en place } \\
\hline Toux & 0 & & 13 & (26) & $<0,001$ \\
\hline Spasme & 2 & (4) & 3 & (6) & $N S$ \\
\hline $\mathrm{SpO}_{2} \leq 85 \%$ & 1 & (2) & 1 & (2) & $N S$ \\
\hline \multicolumn{6}{|l|}{ Retrait } \\
\hline Toux & 0 & & 17 & (34) & $<0,001$ \\
\hline Spasme & 5 & $(10)$ & 17 & (34) & $<0,001$ \\
\hline $\begin{array}{l}\mathrm{SpO}_{2} \leq 85 \% \\
\text { Contamination }\end{array}$ & 1 & (2) & 6 & (12) & $<0,02$ \\
\hline sanguine & 2 & (4) & 15 & (30) & $<0,001$ \\
\hline
\end{tabular}

contre le palais dur, son introduction latérale ${ }^{4,16}$ ou son gonflement partiel, ${ }^{4,17}$ l'utilisation d'un doigt comme guide $^{4}$ ont également été proposés. Dans ces cas plus difficiles, certains auteurs ont employé un laryngoscope, ${ }^{18,19}$ nous y avons en recours une fois au cours de cette étude, mais ceci va un peu à l'encontre des avantages reconnus au ML. ${ }^{13-15}$ Malgré ces difficultés occasionnelles, le MLR n'a pas été plus difficile à poser qu'une sonde endotrachéale.

Lintubation est plus "réflexogène" dans cette série que dans celle des auteurs anglo-saxons ou canadiens, qui ne la réalisent qu'après curarisation, ${ }^{8,20}$ mais elle provoque moins de désaturation. ${ }^{20}$ Néanmoins, une étude anglaise antérieure soulignait déjà la grande inocuité du masque laryngé puisqu'elle ne dénombrait aucun spasme ou stridor chez 20 jeunes patients ayant subi une amygdalectomie. ${ }^{5} \mathrm{Au}$ même stade anesthésique, le masque laryngé est introduit sans réaction, alors que la sonde d'intubation provoque des secousses de toux (26\%). Une anesthésie plus profonde aurait sans doute masqué cette différence. Même si l'introduction du MLR a provoqué une réaction chez trois enfants, insuffisamment endormis, elle n'a pas entrainé de conséquence postopératoire et reste un incident mineur ainsi que l'écrivent de nombreux auteurs. ${ }^{3,8}$ Elle ne semble pas nécessiter le blocage du nerf laryngé supérieur proposé par certains. ${ }^{21}$ Au même stade anesthésique, le retrait du dispositif est plus houleux chez les patients intubés, bien que les enfants soient encore endormis. Cette différence se retrouve également dans la série canadienne, dont les patients sont pourtant extubés au stade chirurgical d'anesthésie et qui rapporte un stridor dans $25,9 \%$ des cas après extubation, et seulement $10 \%$ après retrait du masque laryngé, la fréquence du laryngospasme n'étant pas significativement différente. ${ }^{20}$ 
Lintroduction de l'ouvre-bouche de Boyle-Davis, surtout si la spatule n'a pas été lubrifiée sur sa face en contact avec le tube, peut mobiliser parfois le masque déjà en place, ce qui nécessite sa reposition. ${ }^{7,8}$ Parfois la remise en place de l'ouvre-bouche seul suffit à rétablir la ventilation. L'ouverture de l'ouvre-bouche peut provoquer toux et spasme si l'anesthésie n'est pas assez profonde. ${ }^{20}$ Cet incident ne s'est pas produit dans cette série. Elle peut également entraîner une obstruction respiratoire. Ceci peut se produire lorsque la sonde préformée est écrasée entre la spatule de l'ouvre-bouche et les incisives inférieures. ${ }^{20}$ Le masque laryngé renforcé devrait permettre d'eviter ce genre de problème, mais son armature est plus souple que celle d'une sonde armée et des cas de gêne ventilatoire ont été décrits dans la littérature. ${ }^{22}$ Lorsque l'obstruction ventilatoire est complète, elle est souvent due au glissement du point d'appui de l'ouvre-bouche sur un palais ogival. La spatule devient alors trop longue et déplace le masque laryngé en prenant appui sur le coussinet. Lintubation est souvent la seule solution pour ces enfants, adoptée dans 2 à $4 \%$ des cas selon les séries. ${ }^{8,20}$ Il arrive, lorsque l'enfant présente une petite ouverture du bouche, une macroglossie ou une hypertrophie amygdalienne très importante, que le chirurgien retire et replace l'ouvre-bouche pour l'amygdalectomie controlatérale: Après chaque mise en place de l'ouvrebouche de Boyle-Davis, il est impératif de vérifier que la ventilation se fait librement ${ }^{7}$ en écoutant fonctionner la valve de Digby-Leigh, en regardent les mouvements du ballon ou en suivant le capnogramme. Aucune fuite à l'auscultation du cou ne doit être audible pendant l'insufflation. Le coussinet siliconé ne doit pas être visible dans l'oropharynx, il traduirait un déplacement du masque. Celui-ci peut se produire en raison de l'extrème souplesse du tube et malgré le maintien en position sagittale de la ligne noire. Ce tube armé ne doit pas faire saillie dans l'oropharynx, ce qui correspondrait à l'emploi d'une spatule trop courte. Sonde endotrachéale et masque laryngé sont fermement maintenus dans la rainure de l'abaisse-langue de l'ouvre-bouche qui interdit tout déplacement peropératoire, souvent à l'origine de fuite dans toute autre indication. ${ }^{23}$

Le masque laryngé protège-t-il vraiment les voies aériennes ? La fibroscopie postopératoire semble le prouver dans cette série, tout comme dans d'autres séries ${ }^{8,23}$ malgré quelques traces de sang retrouvées par les auteurs anglo-saxons sur la face laryngée du masque dans un cas sur six. ${ }^{8}$ Cette dernière série décrit en revanche, chez $54 \%$ des enfants intubés avec une sonde préformée, une aspiration hémorragique qui n'est retrouvée que dans $30 \%$ des cas dans notre série. Neuf des 15 enfants qui ont présenté une contamination interne de la sonde ont toussé à l'extubation. Existe-t-il un lien entre ces deux phénomènes, dont l'un pourrait tout à fait favoriser l'autre, l'hémostase obtenue par coagulation restant toujours sujette à caution? Les enfants porteurs dun MLR ont un larynx significativement moins contaminé. Est-ce parce quils n'ont pas toussé ou parce quils sont mieux protégés ? Plusieurs auteurs ont testé l'efficacité de la protection des voies aériennes qu'offrait le masque laryngé à l'aide de produit du contraste ${ }^{14}$ ou de bleu de méthylène, ${ }^{23}$ dont ils ont constaté, par fibroscopie, l'absence de diffusion trachéale. L'étanchéité des voies aériennes protégées par un masque laryngé est tout à fait comparable à celle qu'assure une sonde endotrachéale à ballonnet vis-à-vis de contaminant de provenance endobuccale. ${ }^{14}$

Le masque laryngé renforcé est aussi facile à introduire qu'une sonde endotrachéale. Sa mise en place et son retrait provoquent beaucoup moins d'effets indésirables. L'accès chirurgical est aussi aisé quel que soit le dispositif utilisé. L'assistance ventilatoire sans fuite et surtout l'étanchéité des voies aériennes semblent supérieures à celles obtenues avec une sonde préformée. Le masque laryngé renforcé peut donc être une alternative tout à fait intéressante dans cette indication.

\section{References}

1 Ribeiro OV. Anesthesia for tonsillectomy and adenoidectomy in children: observation in 10000 cases. Anesth Analg 1960; 39: 189-96.

2 Black $A E$, Mackersie AM. Accidental bronchial intubation with RAE tubes. Anaesthesia 1991; 46: 42-3.

3 Alexander CA, Leach $A B$. The laryngeal mask experience of its use in a District General Hospital. Today's anaesthetist 1989; 4: 200-5.

4 Mason $D G$, Bingham $R M$. The laryngeal mask airway in children. Anaesthesia 1990; 45: 760-3.

5 Alexander $C A$. A modified intavent laryngeal mask for ENT and dental anaesthesia (Letter). Anaesthesia 1990; 45; 892-3.

6 Boisson-Bertrand D. Intérêt du "masque laryngé ORL" (Lettre). Ann Fr Anesth Reanim 1992; 11: 119.

7 Boisson-Bertrand D. Le masque larynge "renforcé" dans les adénoamygdalectomies chez l'enfant (Lettre). Ann Fr Anesth Réanim 1993; 12: 82-3.

8 Williams PJ, Bailey PM. Comparison of the reinforced laryngeal mask airway and tracheal intubation for adenotonsillectomy. Br J Anaesth 1993; 70: 30-3.

9 Goodwin APL, Ogg TW. An armored laryngeal mask airway (Letter). Anesthesiology 1992; 76: 150.

10 Wilson IG, Eastley $R$. A modification of the laryngeal mask airway (Letter). Anesthesiology 1991; 74: 1157.

11 Brain AIJ, McGhee TD, McAteer EJ, Thomas A, AbuSaad MAW, Bushmann JA. The laryngeal mask airway. 
Development and preliminary trials of a new type of airway. Anaesthesia 1985; 40; 356-61.

12 Pounder DR, Blackstok D, Steward DJ. Tracheal extubation in children, halothane versus isoflurane, anaesthetized versus awake. Anesthesiology 1991; 74: 653-5.

13 Brodrick PM, Webster NR, Nunn JF. The laryngeal mask airway. A study of 100 patients during spontaneous breathing. Anaesthesia 1989; 44: 238-41.

14 Cork RC, Kaul B, Frink EJ, Standen JR. Comparison of laryngeal mask with endotracheal tube for airway control. Anesthesiology 1991; 75: A 1112.

15 Davies PRF, Tighe SQM, Greenslade GL, Evans DH. Laryngeal mask airway and tracheal tube insertion by unskilled personnel. Lancet 1990; 336: 977-9.

16 Leader $G L$. Facilitation of the insertion of the laryngeal mask (Letter). Anaesthesia 1991; 46: 987.

17 Newman PTF. Insertion of the partially inflated laryngeal mask airway (Letter). Anaesthesia 1991; 46: 235.

18 Lee JJ. Laryngeal mask and trauma to uvula (Letter). Anaesthesia 1989; 44: 1014.

19 Van Heerden PV, Kirrage D. Large tonsils and the laryngeal mask airway (Letter). Anaesthesia 1989; 44: 703.

20 Webster AC, Morley-Forster PK, Dain S, Ganapathy S, Ruby $R$. Anaesthesia for adenotonsillectomy. A comparison between tracheal intubation and the armoured laryngeal mask airway. Can J Anaesth 1993; 40: 1171-7.

21 Dasey N, Mansour $N$. Coughing and laryngospasm with the laryngeal mask (Letter). Anaesthesia 1989; 44: 865.

22 Heath $M L$, Sinnathamby $S W$. The reinforced laryngeal mask airway for adenotonsillectomy (Letter). $\mathrm{Br} \mathrm{J}$ Anaesth 1994; 71: 728-9.

23 John RE, Hill S, Hugues TJ. Airway protection by the laryngeal mask. A barrier to dye placed in the pharynx. Anaesthesia 1991, 46: 366-7. 